\title{
A Full-wave Rectifier Based on Memristive Systems
}

\author{
Jiri Vavra and Dalibor Biolek \\ Department of Electrical Engineering, Department of Microelectronics \\ University of Defence, Brno University of Technology \\ Brno, Czech Republic \\ \{jiri.vavra, dalibor.biolek\}@unob.cz
}

\begin{abstract}
Precision low-voltage rectifiers are important building blocks for analog signal processing. In this paper, a full-wave rectifier based on two Bipolar Memristive Systems with the Threshold and one inverter is presented. The traditional approach based on OpAmp and diodes exhibits undesirable effects caused by diode commutations and OpAmp imperfections. The PSpice simulation results are included.
\end{abstract}

Keywords-Memristive System, Full-Wave Rectifier.

\section{INTRODUCTION}

In recent years, the number of papers devoted to applications of memristive systems for analog signal processing is permanently increasing. It follows from the analysis of the extensive database of the references devoted to memristors and other memory elements [1]. Many designs start from the traditional analog solutions. At the final stage, the internal resistor is subsequently replaced by its memory version, thus the memristor [2-4]. It opens-up the possibility of an elegant electronic control of the application. In fact, the authors do not discuss the unavoidable signal distortion, the offset and other issues which follow directly from the principle of the memristor [3], [4]. Other problems are connected with the confusions generated by the disinterpretation of the behavior of real memristive systems which exhibit - in contradiction to the frequently used simple model of the HP memristor [5], the threshold property and much more complicated dynamic behavior. As a result, some of the suggested analog applications of memristors can bring more disadvantages rather than being innovative and beneficial, and their practical workability beyond the SPICE environment is rather problematic.

The professional synthesis of memristor-based applications would benefit from the unique characteristics of memristive systems. However, such solutions are rare [6-10]. Some of them are summarized in [11].

Different applications require different characteristics of memristive systems. For example, memory and logic applications need the elements not only for the calculation and control, but also the ability to store data after the calculation. Reading and writing the data in these elements should be done sufficiently fast; moreover, the readout mechanism must be non-destructive for stored data. To store a known digital state in nonvolatile way and concurrently maintain a low sensitivity to the parameter variation and changing the operating conditions, the noise immunity, the stand-by as well as transient read-write power consumptions may be critical issues.

In analog applications, the characteristics of memristors used in the design strongly depend on the regime of the operation (linear, nonlinear, fixed or adjustable parameters, (non)volatility requirements, etc.).

The analysis given below starts from the paper [11], which specifies the requirements for the characteristics of a memristive system which would be useful for its practical utilization in various areas of the analog signal processing. The bipolar memristive system with the voltage threshold (BMST) [12-14] is selected as a useful type for a signal rectification. It is shown that, when properly selecting its parameters, it behaves as a low-voltage diode. Simple fullhalf memristor-based rectifier is proposed, and its operation is verified via SPICE simulation. In contrast to the memristive rectifier proposed in [15], which resembles a classical Graetz bridge rectifier, our circuit employs two memristive elements and one voltage inverter.

\section{MEMRISTIVE SYSTEMS FOR NONLINEAR CIRCUITS}

The ideal voltage-controlled memristor as the fourth fundamental circuit element [16] is defined as a twoterminal device with port and state equations

$$
\begin{aligned}
& i=g(x) v \\
& \frac{d}{d t} x=v
\end{aligned}
$$

Where $i$ and $v$ are the memristor current and voltage, $x$ is a state variable, and $g$ is a function, nonlinear in general, modelling the memductance as a function of the state. It is obvious from the differential equation (2) that the state variable $x$ is in fact the integral of voltage, denoted as flux.

However, designing practically operating devices utilizing the memristors must take into account two factors: 1) Some of the ideal parameters of the memristor modeled via the above equations would bring essential practical problems (especially the issue of infinite memory depth" [17] and the ability of integrating, thus amplifying any offset). 2) Today's manufactured specimen of various memristive devices exhibit the behavior that cannot be described by such primitive models. For the purposes of designing memristor-based rectifiers, the above problems can be partially solved via utilizing the so-called Bipolar Memristive system with a Threshold (BMST), or via similar 
memristive system based on BMST, acting as nonlinear resistor with the threshold voltage decreased as much as possible towards zero.

The simplest BMST model, useful for such purposes, can be described by the following modified port and state equations:

$$
\begin{gathered}
i=R_{M}^{-1} v \\
\frac{d}{d t} R_{M}=f_{L}(v)
\end{gathered}
$$

The port equation (3) defines the state-dependent Ohm's law between the terminal voltage and current. The memristance $R_{M}$ serves as the state variable whose derivative with respect to time depends, according to (4), on the product of nonlinear function $f_{L}$. The function $f_{L}$ shown in Fig. 1 depends on the terminal voltage, introducing the voltage threshold property into the behavior of the element. The corresponding threshold limits $V_{t-}$ and $V_{t^{+}}$provide the offset and drift immunity. Its values also affect the measure of the nonlinear operation of this device, which can be different for concrete application. For low-voltage rectification, the voltage threshold levels should be as low as possible.

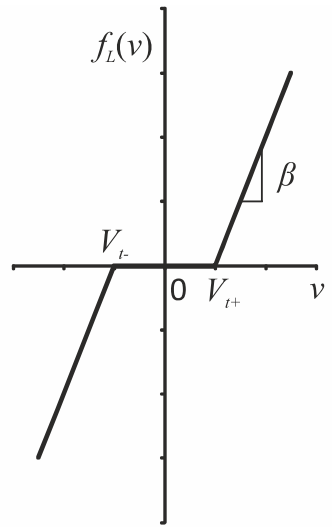

Figure 1. Example of graph of function $f_{L}$ [11].

The above-threshold region is characterized by the slope $\beta\left[\mathrm{A}^{-1} \mathrm{~s}^{-1}\right]$ of $f_{L}(v)$. It is obvious from Eq. (4) that the parameter $\beta$ controls the speed of developing the $R_{M}$, thus the system dynamic behavior in the transitional regime.

However, the above model does not introduce the $R_{M}$ limitation by saturating states $R_{o n}$ and $R_{o f f}$. For relative high values of $\beta$ (in hundreds of $\mathrm{GA}^{-1} \mathrm{~s}^{-1}$ ), the system operates practically in a bistable regime within two limit states $\mathrm{ON}$ and OFF. In ON (OFF) state, the internal memristance $R_{M}$ is relatively low (high). The above limitation is modeled via the window function $w\left(R_{M}, v\right)$ in the modified state equation

$$
\frac{d}{d t} R_{M}=f_{L}(v) w\left(R_{M}, v\right)
$$
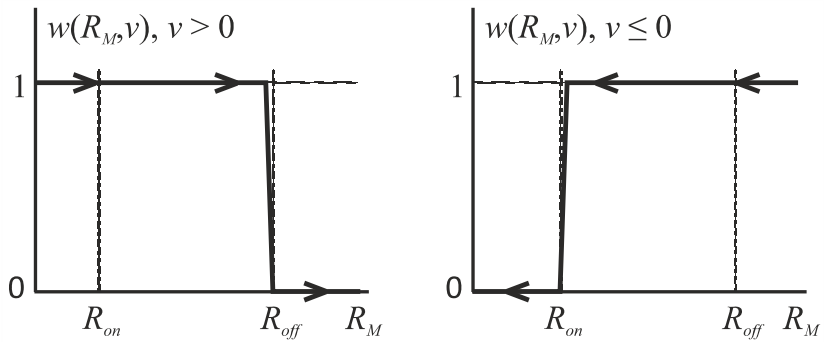

Figure 2. Graph of Biolek window function $w$ [11].

The mechanism of such a windowing is illustrated in Fig. 2. Switching from one state to another is dependent on the terminal voltage of BMST. For positive voltage above the threshold value, the window function is 1 for memristance below $R_{o f f}$, thus the $R_{M}$ derivative is positive according to Eq. (5), and it increases towards its upper limit $R_{\text {off. }}$. In the proximity of this boundary the window function switches its value to zero and the memristance stops moving. If the voltage changes its sign, the operation continues analogously, according to the curve on the right side of Fig. 2, towards the other limiting state $R_{o n}$.

The above window function, published in rather different form by Biolek et al in [18], can be described by the following formula:

$$
w\left(R_{M}, v\right)=\theta(v) \theta\left(R_{o f f}-R_{M}\right)+\theta(-v) \theta\left(R_{M}-R_{o n}\right)
$$

Where denotes the unity step function.

The window function from Eq. (6) has been used hereinafter for SPICE simulations. This window works reliable in the SPICE environment and it also prevents the well-known "stick effect" at boundaries.

The results of the SPICE DC analysis of the BMST device is shown in Fig. 3 in the form of the current-voltage DC plot $I_{M}$ vs. $V_{M}$. The following parameters were used for the simulation: $R_{o n}=100 \Omega, R_{o f f}=100 \mathrm{k} \Omega, \beta=10^{14} \mathrm{~A}^{-1} \mathrm{~s}^{-1}$, $V_{t}=0.1 \mathrm{~V}$.

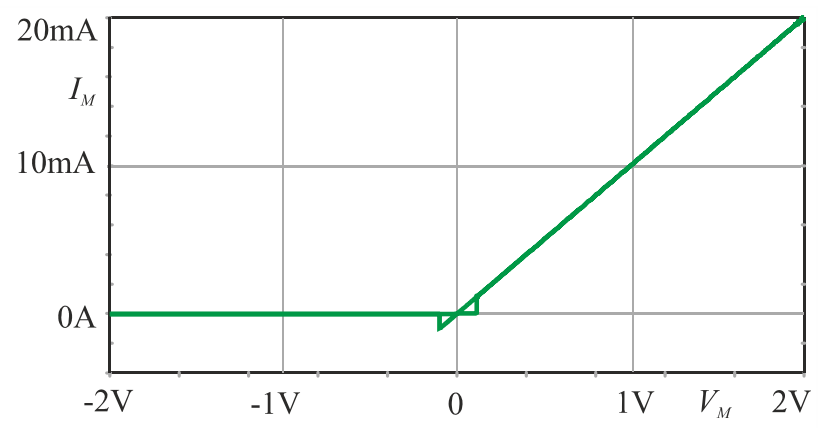

Figure 3. Current-voltage characteristic of BMST for $V_{t}=100 \mathrm{mV}$.

A typical "butterfly" effect around the $v-i$ origin can be observed, caused by the effect of nonzero threshold levels (here $0.1 \mathrm{~V}$ ). After switching the memristive device into its boundary state, it behaves as linear resistor. It is obvious that for such a case, the BMST acts as a two-state linear resistor whose state switches between either states depending on the 
polarity of the terminal voltage. For $R_{o f f} \gg>R_{o n}$, it can be used as a diode.

The threshold effect of the current-voltage characteristic in Fig. 3 can be smoothed away after decreasing the threshold levels, for example to $V_{t}=1 \mathrm{mV}$. The corresponding simulation results are shown in Fig. 4.

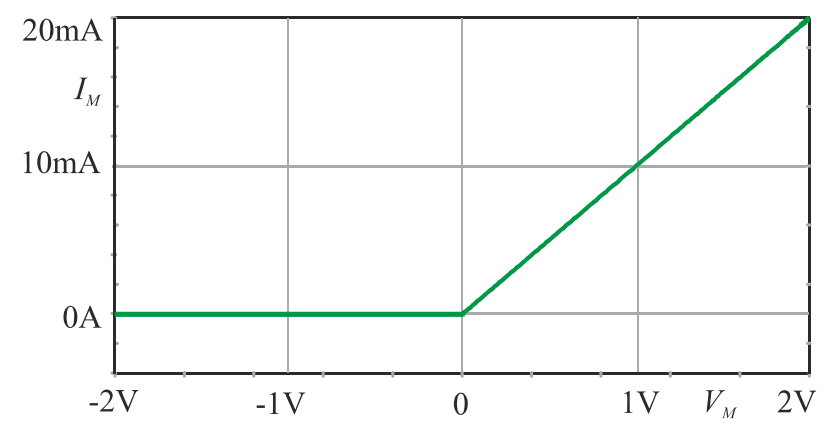

Figure 4. Current-voltage characteristic of BMST for $V_{t}=1 \mathrm{mV}$.

The limiting parameters for such a design are nonzero threshold voltage, nonzero memristance in ON-state, $R_{o f f} / R_{o n}$ ratio, and finite switching time between both states which limits the rectifier dynamics.

According to the belief of the authors, the memristive systems, BMST being of one of them, will be manufactured with their prescribed characteristics in the near future. For the purpose of the simulations, concrete parameters, specified below, have been used in order to optimize the performance of the rectifier.

\section{Full-WAVE MEMSYSTEM-BASED RECTIFIER}

The proposed full-wave rectifier in Fig. 5 employs two BMST operating in two states $\mathrm{ON}$ and OFF, modeled by Eqs. (3), (5), and (6), with a careful selection of the crucial parameters of the model, particularly the threshold voltage $V_{t}$ and the slope $\beta$. As shown in Fig. 5, in addition to two memristors, the circuit utilizes only one simple voltage inverter. This brings an advantage of a high dynamic range of the operation.

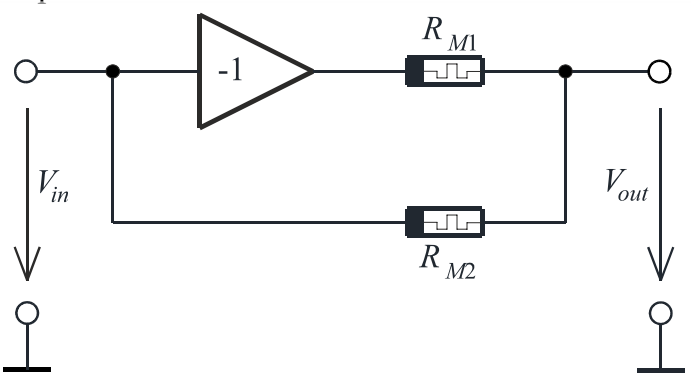

Figure 5. Full-wave memsystem-based rectifier.

Both BMSTs "sharply" change their internal memristances between $R_{o n}$ and $R_{\text {off }}$ depending on the polarities of their terminal voltages. The voltage inverter provides the required voltage for the BMST $R_{M 1}$. The circuit operates on the principle of MAX circuitry, for the first time described in [7], and also applied in [11] and [15]. Due to anti-series interconnection of the BMSTs, the current flowing through enforces to switch these devices always into two different states. If positive voltage appears at the input, the inverter supplies the BMST $R_{M 1}$ by its inverted copy, and the $R_{M 2}\left(R_{M 1}\right)$ memristor gets to the ON (OFF) state. As a result, the output voltage will be practically equal to the input voltage. For a negative polarity of the input voltage, the states of the BMST are interchanged, and the output voltage will be given by the output voltage of the inverter.

The parameters of both memristive devices were selected as follows: the BMSTs are identical with symmetric $f_{L}$ function, with the threshold voltage $V_{t}=1 \mathrm{mV}$, and with the slope $\beta=10^{14} \mathrm{~A}^{-1} \mathrm{~s}^{-1}$. The limiting values of memristances $R_{\text {on }}=1 \Omega, R_{\text {off }}=1 \mathrm{M} \Omega$. High slope of $f_{L}$ function above the threshold voltage ensures a fast switching between limiting $\mathrm{ON}$ and OFF states. The result of the SPICE transient analysis of the proposed circuit for $5 \mathrm{~V} / 10 \mathrm{kHz}$ sinusoidal excitation is shown in Fig. 6.

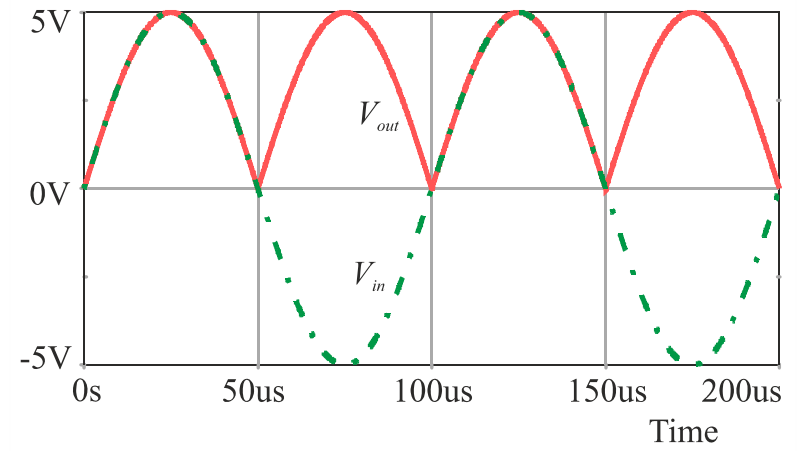

Figure 6. Transient analysis of the proposed full-wave memrectifier with $V_{t}=1 \mathrm{mV}$.

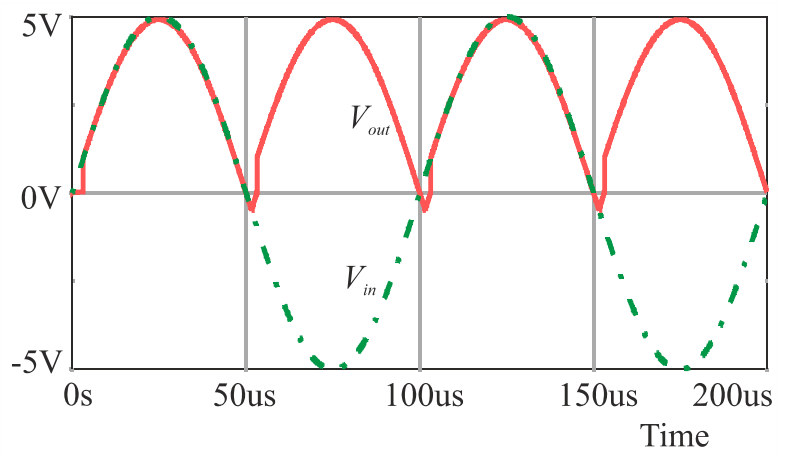

Figure 7. Transient analysis of proposed full-wave memrectifier with $V_{t}=$ $100 \mathrm{mV}$.

It should be noted that, in comparison to the classical ideal memristor, the advantage of the BMST consists in its insensitivity to the initial state of the memristance. This fact was also verified via the simulation.

As already mentioned above, the threshold voltage $V_{t}$ plays an important role for the mem-rectifier performance. The threshold levels should be symmetrical $\left(V_{t^{+}}=-V_{t-}=V_{t}\right)$ and as small as possible. It is necessary to find a compromise between the accuracy of rectifying low-level 
signals and the memelement immunity to the ubiquitous offset and drift. The transient analysis in Fig. 7 of the same circuit but with a higher threshold voltage of both BMST, specifically $V_{t}=0.1 \mathrm{~V}$, demonstrate that the distortion of the output signal around zero considerably increases.

\section{CONCLUSSION}

It is shown in the paper that the memristive device of BMST type (Bipolar Memristive System with Threshold) can be a promising building block for nonlinear analog applications. In the case of its fabrication as a nano-device, it can be also advantageously used for low-voltage ultra-lowpower designs. It is important that five device parameters, namely $R_{o n}, R_{o f f}, V_{t^{+}}, V_{t-}$, and $\beta$, can be used for accommodation of this block for its optimum performance in various applications. For a specific set of these parameters, the two BMSTs together with one voltage inverter can serve as a full-wave rectifier. In addition to the classical OpAmpbased rectifiers, its accuracy and speed can be optimized independently via tweaking the threshold voltage, $R_{\text {on }}, R_{\text {off }}$ (accuracy) and the slope $\beta$ (speed).

\section{ACKNOWLEDGMENT}

The research is a part of the COST Action IC1103 and it is financially supported by the Czech Ministry of Education under grant no. LD14103. The research was also supported by the Project for the development of K217 Department, UD Brno.

\section{REFERENCES}

[1] http://memlinks.eu/ - interactive database of papers dealing with memory elements.

[2] Y. V. Pershin and M. Di Ventra, "Practical Approach to Programmable Analog Circuits With Memristors," IEEE Trans. on Circuits and Systems I: Regular Papers, vol. 57, no. 8, pp. 1857-1864, 2010

[3] A. Ascoli, R. Tetzlaff, F. Corinto, M. Mirchev, and M. Gilli, "Memristor-based filtering applications," In: Test Workshop (LATW), 2013 14th Latin American, 2013, pp. 1-6.

[4] A. Talukdar, A.G. Radwan, and K.N. Salama, "Non linear dynamics of memristor based 3rd order oscillatory system," Microelectronics Journal, vol. 43, no. 3, pp. 169-175, 2012.
[5] D. B. Strukov, G. S. Snider, D. R. Stewart, and R. S. Williams, "The missing memristor found," Nature, vol. 453, 2008, pp. 80-83.

[6] I. C. Goknar, F. Öncül, and E. Minayi, "New Memristor Applications: AM, ASK, FSK, and BPSK Modulators," Antennas and Propagation Magazine, IEEE, vol. 55, no. 2, pp. 304-313, 2013.

[7] M. Klimo and O. Šuch, "Memristors can implement fuzzy logic," arXiv:1110.2074v1, [cs.ET], 2011

[8] R. Berdan, C. Toumazou, and T. Prodromakis, "High precision analogue memristor state tuning," Electronics Letters, vol. 48, no. 18, pp. 1105-1107, 2012.

[9] M. Mahvash and A.C. Parker, "A memristor SPICE model for designing memristor circuits," In: 2010 53rd IEEE International Midwest Symposium on Circuits and Systems (MWSCAS), pp. 989992, 2010.

[10] M.A. Zidan, H. Omran, C. Smith, A. Sayed, A.G. Radwan, and K.N. Salama, "A family of memristor-based reactance-less oscillators," Int. J. of Circ Theory and Applications, vol. 42, no. 11, pp. 1103-1122, 2013.

[11] D. Biolek, V. Biolková, and Z. Kolka, "Memristive Systems For Analog Signal Processing," In: 2014 IEEE International Symposium on Circuits and Systems (ISCAS 2014), Melbourne, Australia, 2014, pp. 2588-2591.

[12] Y. V. Pershin and M. Di Ventra, "Experimental demonstration of associative memory with memristive neural networks," Neural Networks, vol. 23, no. 7, pp. 881-886, 2010.

[13] D. Biolek, M. Di Ventra, and Y.V. Pershin, "Reliable SPICE Simulations of Memristors, Memcapacitors and Meminductors," Radioengineering, vol. 22, no. 4, pp. 945-968, 2013. ISSN 12102512 .

[14] S. Kvatinsky, E. G. Friedman, A. Kolodny, and U. C. Weiser, "TEAM: ThrEshold Adaptive Memristor Model," IEEE Trans. on Circuits and Systems I: Regular Papers, vol. 60, no. 1, pp. 211-221, 2013.

[15] O. Pabst and T. Schmidt, "Frequency dependen trectifier memristor bridge used as a programmable synaptic membrane voltage generator," Journal of Electrical Bioimpedance, vol. 4, pp. 23-32, 2013.

[16] L. O. Chua, "Memristor - The Missing Circuit Element," IEEE Trans. Circuit Theory, vol. CT-18, no. 5, pp. 507-519, Sept. 1971.

[17] D. Biolek, Z. Biolek, V. Biolková, and Z. Kolka, "Some Fingerprints of Ideal Memristors," In: 2013 IEEE International Symposium on Circuits and Systems (ISCAS 2013). Beijing, China: IEEE, 2013, p. 201-204. ISBN 978-1-4673-5761-6.

[18] Z. Biolek, D. Biolek, and V. Biolková, "SPICE model of memristor with nonlinear dopant drift," Radioengineering, vol. 18, no. 2, Part II, pp. 210-214, June 2009. ISSN 1210-2512. 